\title{
Implementation Intentions and Shielding Goal Striving From Unwanted Thoughts and Feelings
}

\author{
Anja Achtziger \\ Universität Konstanz, Germany \\ Peter M. Gollwitzer \\ Universität Konstanz, Germany, and New York University, USA \\ Paschal Sheeran \\ University of Sheffield, United Kingdom
}

Forming an implementation intention or "if-then plan" promotes the attainment of different types of goals (Gollwitzer, 1999; Gollwitzer \& Sheeran, 2006). So far, research on implementation intentions has focused on the initiation of goal striving, whereas the issue of shielding of ongoing goal striving has been largely neglected. In two field experiments concerned with dieting (Study 1) and athletic goals (Study 2), goal shielding was supported by implementation intentions geared at controlling potentially interfering inner states (i.e., cravings for junk food in Study 1, and disruptive thoughts, feelings, and physiological states in Study 2). In both experiments, forming ifthen plans enhanced the rate of goal attainment. Thus, implementation intention formation can be used to promote the realization of desired outcomes not only by facilitating getting started with goal striving but also by preventing goal striving from straying off course.

Keywords: implementation intentions; goal achievement; goal shielding; dieting; sport

G ollwitzer $(1993,1999)$ differentiates two kinds of intentions: goal intentions and implementation intentions. Goal intentions are defined as end states an individual wants to attain; they turn desires into binding goals. Therefore, goal intentions can be thought of as goals in the common sense as they have the format of "I want to attain X!" (e.g., "I want to eat healthily!"). Implementation intentions are formed to support the realization of goal intentions by specifying when, where, and how goal-directed responses should be initiated. Implementation intentions have the format of if-then plans: "If situation $\mathrm{X}$ arises, then I will do Y!" (Gollwitzer, 1993, 1999). Thus, in the case of the goal intention "I want to eat healthily!" a supporting if-then plan could be "If I order something to eat in my favorite restaurant, then I will take a vegetarian meal!”

Implementation intentions create a mental link between a selected cue or situation (e.g., the occurrence of a certain stimulus such as the number 3 on a computer screen) and a goal-directed response (e.g., pressing a response key as fast as possible; Brandstätter, Lengfelder, \& Gollwitzer, 2001). Forming an implementation intention commits one to perform this goaldirected response as soon as the specified situation is encountered. For instance, Bayer and Gollwitzer (2007, Study 1) observed that female high school students performed better in a mathematics test if they supported

Authors' Note: We thank the Center for Research on Intentions and Intentionality (Universität Konstanz) for funding this research and Norbert Michalski for the acquisition of the data for Study 2. Moreover, we thank two anonymous reviewers for their valuable comments on an earlier version of this article. Please address correspondence to Anja Achtziger, Universität Konstanz, Universitätsstr. 10, D-78457 Konstanz, Germany; phone: +49-7531-883287; fax: +497531-883286; e-mail address: anja.achtziger@uni-konstanz.de. 
their goal intention "I will correctly solve as many problems as possible!" with the implementation intention "And if I start a new problem, then I will tell myself: I can solve this problem!" As a consequence of forming this implementation intention, a strong mental link is created between the external situation of starting a new problem on the test and the goal-directed response of motivating oneself with respect to performing well on the mathematics test.

The mental link created by an implementation intention is expected to facilitate goal attainment on the basis of the following psychological processes: By forming an implementation intention, the mental representation of the specified cue becomes highly activated and therefore more easily accessible (Gollwitzer, 1999). This heightened cognitive accessibility makes it easier to detect the critical cue in relevant situational contexts and to readily attend to it even if one is busy with other things. Moreover, because of its heightened cognitive accessibility, the recall of the specified situation is facilitated. The hypothesis of a heightened cognitive accessibility of the situation specified in the if-component of the implementation intention is supported by many studies (e.g., Aarts, Dijksterhuis \& Midden, 1999; Webb \& Sheeran, 2004). For instance, Achtziger, Bayer, and Gollwitzer (2007) observed that participants holding implementation intentions attended to and recalled the situation specified in an implementation better than did participants who only hold mere goal intentions. Next to the heightened cognitive accessibility of the cue specified in the implementation intention, automatic initiation of the response specified in the then-component was also found to be responsible for the effectiveness of implementation intentions. In the presence of the critical cue, the respective response is executed immediately (Gollwitzer \& Brandstätter, 1997), without further conscious intent (Bayer, Achtziger, Malzacher, Moskowitz \& Gollwitzer, 2007), and efficiently (Brandstätter et al., 2001).

In the past 10 years, research in different fields of psychology showed that forming an implementation intention by specifying an anticipated cue as a condition for initiating goal-directed responses leads to more frequent goal attainment compared to setting mere goal intentions. This is especially true when goal intentions were hard to attain or somewhat unpleasant to act on (reviews by Achtziger \& Gollwitzer, 2008; Gollwitzer \& Sheeran, 2006). Most of these studies investigated the self-regulatory issue of getting started with goal striving by executing relevant goal-directed responses. Accordingly, the implementation intentions under study specified a good opportunity to act in the if-component and an appropriate instrumental goal-directed response in the thencomponent of the if-then plan. However, as soon as a person has succeeded with initiating goal striving, it still needs to be brought to a successful ending to guarantee goal attainment. For this purpose, people need to protect ongoing goal striving from getting derailed. This becomes a particularly pressing issue when the individual faces the following impeding conditions (Gollwitzer \& Sheeran, 2006; Gollwitzer, Bayer, \& McCulloch, 2005): (a) conflicting attention and behavioral responses that make people stray off course (e.g., distractions and temptations), (b) obstacles and barriers that are not anticipated by the individual (e.g., deindividuating performance contexts may lead to social loafing), (c) detrimental inner states that threaten goal attainment (e.g., fears, moods, ego depletion), and (d) situationally activated antagonistic goals that produce goal conflicts.

The present research investigates whether people can use implementation intentions to protect goal striving in one such critical situation (i.e., detrimental inner states). Specifically, we tested whether detrimental inner states such as disruptive thoughts (i.e., cravings) and feelings (i.e., performance anxiety) can be controlled by implementation intentions so that goal striving can proceed undisturbed. Such implementation intentions would have to specify a potentially disruptive inner state in the if-component and link it to an instrumental coping response in the then-component. By facilitating the execution of the coping response specified in the thencomponent, such implementation intentions are expected to effectively shield the ongoing goal striving from getting derailed by the detrimental inner state specified in the if-component.

\section{INNER STATES AND GOAL SHIELDING}

Certain inner states are known to strongly imperil goal attainment. For instance, Herman and Polivy (2004) showed that desire-related thoughts (cravings) for unhealthy foodstuffs imperiled the goal of self-regulating eating behavior. Williams, Vickers, and Rodrigues (2002) observed that performance anxiety led to decrements in efficiency and effectiveness during table tennis training. Accordingly, we investigated whether these inner states (i.e., cravings and fear) can be specified in the if-component of implementation intentions and be linked to an effective shielding response in the then-part. If people could make use of these inner states as initiators of goal-shielding responses, they would have a self-regulating strategy at their disposal that could be used across all kinds of goal strivings alike as well as across various disruptive situational contexts. One does not need to anticipate what kind of external disruptive event will ultimately threaten one's goal striving at hand to form a protective implementation intention. Rather, the disruptive inner states (e.g., performance anxiety) generated by these negative 
events can be used as a summary label and placed in the if-component of a protective implementation intention.

To investigate this possibility, we ran two studies, using disruptive inner states as if-components of protective implementation intentions: Study 1 investigated whether specifying the negative inner state of craving for high-fat food in the if-component of an implementation intention and linking it to an ignore response can protect striving toward the goal of eating healthily. Study 2 investigated whether implementation intentions to ignore negative inner states of irritation and performance anxiety can be used to shield the goal of performing well in tennis competitions.

\section{STUDY 1: CONTROLLING CRAVINGS BY MEANS OF IMPLEMENTATION INTENTIONS}

Research indicates that people's efforts to control their food intake are plagued by frequent failure (e.g., Garner \& Wooley, 1991; Heatherton, Mahamedi, Striepe, Field, \& Keel, 1997). A key factor in such self-regulatory failures are desire thoughts (cravings) for unhealthy foodstuffs that overwhelm people's good intentions (e.g., Herman \& Polivy, 2004; Kavanagh, Andrade, \& May, 2005; Loewenstein, 1996). The elaborated intrusion theory of desire (EITD; Kavanagh et al., 2005) proposes that the intrusive effects of desire result from the interplay of automatic and deliberative processes. External cues trigger spontaneous thoughts about the target that are perceived as intrusive. These intrusive thoughts are the result of learned cue-target associations and place little demand on cognitive resources. However, when the intrusive thoughts elicit a strong affective reaction or provoke the experience of deprivation, then the thought will become elaborated, which requires controlled processes. Progressive elaboration of the target-related thoughts is the mechanism that explains the persistence and influence of desire. Elaboration alters the prioritization of attentional and working memory processes, making it likely that additional internal/external cues are accessed and that further intrusive thoughts are generated. The processing priority that is accorded to elaborating thoughts about the target explains why progress toward superordinate goals (e.g., the dieting goal) often is undermined during craving episodes.

The distinction between intrusive and elaborated thoughts has important implications for efforts to control desire. If people do not attend to intrusive thoughts or do not retain those thoughts in working memory, then elaboration of desire-relevant information should be prevented, and thus goal striving should not get disrupted. Study 1 tested this idea. Participants were asked about their goal intentions to reduce consumption of a high-fat snack food. In addition, a subset of participants was asked to form an implementation intention to ignore thoughts about that foodstuff. The prediction tested is that participants who form an if-then plan to shield their dieting goal from unwanted food cravings will show greater reduction in snack food consumption compared with control participants who do not form an if-then plan.

\section{Method}

\section{Participants and Design}

Undergraduates at a U.K. university $(N=92 ; 74$ women and 18 men; $M$ age $=19.50, S D=3.79$ ) took part in a study on snack food consumption. Participants were randomly assigned to one of two conditions (control vs. implementation intention).

\section{Procedure}

Baseline questionnaire. Participants completed a baseline questionnaire that asked them to specify the following: "Which high-fat food (e.g., chocolate, pizza, French fries) have you eaten most of in the last week?" and "How many times did you consume this food in the last week?" Several other variables were assessed based on Ajzen's (1991) theory of planned behavior. All answer scales ranged from 1 to 7 . Goal intention was measured by "I intend to halve my consumption of this food in the next week" (definitely no-definitely yes) and "I will try to halve my consumption of this food in the next week" (definitely no-definitely yes) $(\alpha=.92)$. Perceived control was measured by the following: "For me to halve my consumption of this food in the next week would be ..." (easy-difficult), "I am confident I can halve my consumption of this food in the next week" (strongly disagree-strongly agree), and "How certain are you that you can halve your consumption of this food in the next week?" (very uncertain-very certain) $(\alpha=.82)$. Subjective norm was measured by a single item: "Most people who are important to me think that I should halve my consumption of this food in the next week" (definitely no-definitely yes). Attitudes were measured by responses to the stem, "For me to halve my consumption of this food in the next week would be ..." on five bipolar scales (pleasant-unpleasant, healthy-unhealthy, important-unimportant, enjoyableunenjoyable, satisfying-unsatisfying) $(\alpha=.77)$. Finally, social desirability was measured using the 10 -item version of the Marlowe-Crowne scale (Crowne \& Marlowe, 1960) developed by Strahan and Gerbasi (1972). Reliability was modest among the present sample $(\alpha=.47)$.

Implementation intention manipulation. The implementation intention manipulation came right after the 
assessment of social desirability. Participants were asked, "Please tell yourself: 'And if I think about my chosen food, then I will ignore that thought!' Please say this line to yourself three times and commit yourself to acting on it. When you have said the line to yourself three times, tick this box." In the control condition, participants received no instructions to form implementation intentions after they had completed the social desirability items.

Follow-up questionnaire. One week later, all participants completed a follow-up questionnaire asking for the number of times they had consumed their specified foodstuff during the previous week.

\section{Results}

\section{Equivalence of Conditions}

We tested whether implementation intention participants differed from control participants on background variables. A multivariate analysis of variance (MANOVA) on the variables age, goal intention, perceived control, attitude, norm, past consumption, and social desirability showed no overall effect of condition, $F(7,84)=$ $0.63, n s\left(\eta^{2}=.05\right)$, and none of the univariate tests were significant. Chi-square tests showed equivalent proportions of men and women in the two conditions and that both conditions specified equivalent foodstuffs ( $p s>$ .13). Social-desirability scores were not associated with reported snack food consumption at follow-up among implementation intention or control participants $(r s=-.10$ and .03, respectively, $p s>.47)$.

\section{Impact of Implementation Intention Formation on Reducing Consumption}

To test whether implementation intention formation reduced consumption of specified high-fat snacks, we conducted a 2 (Implementation Intention: Formed vs. Not Formed) $\times 2$ (Time: Baseline vs. Follow-up) repeated measures ANOVA. The main effect of Implementation Intention was not significant, $F(1,90)=0.71$, ns $\left(\eta^{2}=\right.$ .001). There was a highly significant main effect of Time, $F(1,90)=27.99, p<.001\left(\eta^{2}=.24\right)$, such that all participants reduced their consumption of snack food from baseline to follow-up ( $M s=4.17$ and $2.96, S D s=2.47$ and 2.49 , respectively). This main effect was qualified by the expected interaction between Implementation Intention and Time, $F(1,90)=3.86, p=.05\left(\eta^{2}=.04\right)$. Figure 1 presents the mean number of snacks consumed by the groups at both time points. Planned comparisons showed that the implementation intention and control groups did not differ on either baseline or follow-up consumption, $F$ s $<1.16$. Moreover, there was a significant reduction in consumption

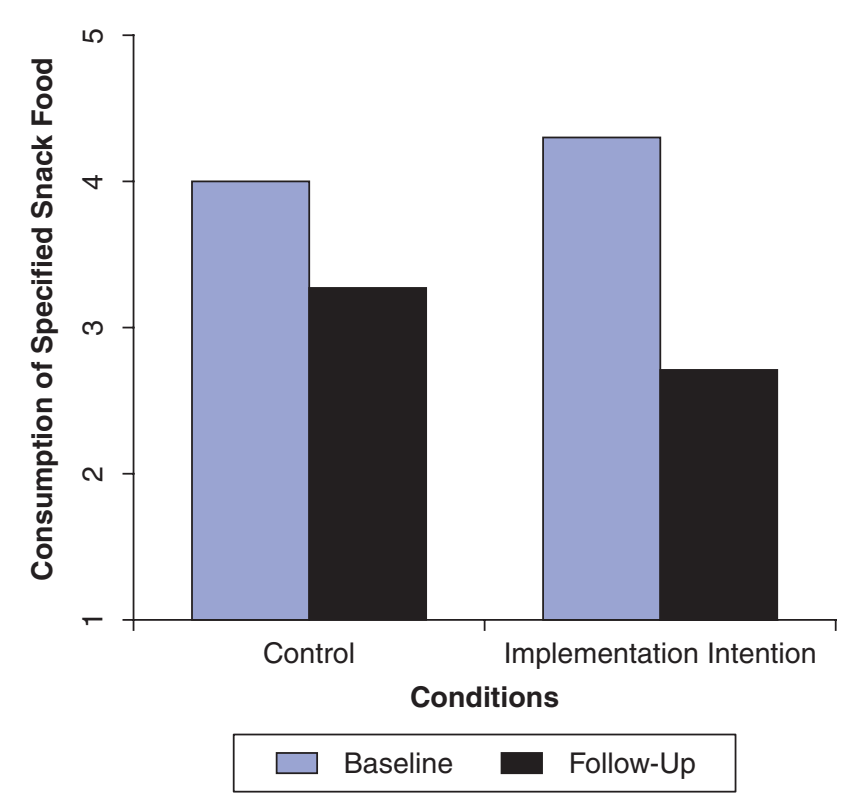

Figure 1 Consumption of specified snack food at baseline and follow-up by condition.

among control participants, $F(1,40)=3.99, p=.05\left(\eta^{2}=\right.$ $.09)$, and participants who formed implementation intentions, $F(1,50)=36.92, p<.001\left(\eta^{2}=.43\right)$. Thus, the interaction is explained by the significantly greater reduction in snack food consumption among implementation intention participants $(M=-1.60, S D=1.88)$ as compared to control participants $(M=-0.73, S D=2.35)$.

\section{Impact of Implementation Intention Formation on Goal Attainment}

We also examined to what extent implementation intention versus control participants succeeded in reaching the goal of halving their consumption of chosen snack foods. One-sample $t$ tests showed that control participants consumed significantly more snacks at follow-up ( $M=3.27)$ compared to one-half of their baseline consumption $(M=2.00), t(40)=3.21, p<$ .001. In contrast, there was no significant difference between half of baseline consumption $(M=2.15)$ and consumption at follow-up $(M=2.71)$ among participants who formed implementation intentions, $t(40)=$ $1.62, p>.11$. Thus, participants who formed if-then plans achieved their goal, unlike participants with equivalent goal intentions who did not plan.

\section{Moderated Regression Analyses}

Participants in the present study generally had weak goal intentions to halve their consumption of high-fat snacks $(M=3.60, S D=1.82)$; in fact, $54.3 \%$ of the sample scored below the midpoint (4) on the intention scale. 
TABLE 1: Regression of Snack Food Consumption at Follow-Up on Predictor Variables (Study 1)

\begin{tabular}{|c|c|c|c|c|c|}
\hline \multirow[b]{2}{*}{ Step } & \multirow[b]{2}{*}{ Variable Entered } & \multicolumn{4}{|c|}{ Betas } \\
\hline & & Step 1 & Step 2 & Step 3 & Step 4 \\
\hline \multirow[t]{2}{*}{1.} & Baseline consumption & $.64 * * *$ & $.57 * * *$ & $.59 * * *$ & $.59 * * *$ \\
\hline & Social desirability & -.10 & -.05 & -.04 & -.04 \\
\hline \multirow[t]{4}{*}{2.} & Goal intention & & -.11 & -.12 & .11 \\
\hline & Attitude & & -.04 & -.04 & -.05 \\
\hline & Subjective norm & & .10 & .08 & .08 \\
\hline & Perceived control & & -.11 & -.09 & -.08 \\
\hline 3. & If-then plan & & & $-.15^{\dagger}$ & $-.15^{\dagger}$ \\
\hline \multirow[t]{5}{*}{4.} & Goal intention $\times$ If-Then Plan & & & & $.31 * *$ \\
\hline & $R^{2}$ & .41 & .44 & .46 & .50 \\
\hline & Model $F$ & $30.26 * *$ & $10.76 * * *$ & $9.90 * * *$ & $10.23 * *$ \\
\hline & $\Delta R^{2}$ & - & .03 & .02 & .04 \\
\hline & $\Delta F$ & - & 1.01 & $3.08+$ & $7.25^{* *}$ \\
\hline
\end{tabular}

NOTE: ${ }^{\dagger} p<.09 .{ }^{* *} p<.01 .{ }^{* * *} p<.001$.

Because strong implementation intention effects are only expected to emerge when participants hold strong goal intentions (e.g., Sheeran, Webb, \& Gollwitzer, 2005), we conducted a moderated regression analysis to test whether implementation intention formation especially benefited participants who strongly intended to reduce their consumption of high-fat snacks. Snack food consumption at follow-up was regressed on past consumption and social desirability at Step 1, the strength of goal intention and other theory of planned behavior variables at Step 2, and dummy-coded implementation intention (not formed $=0$, formed $=1$ ) at Step 3. Goal intention scores were standardized before computing the Goal Intention Strength $\times$ Implementation Intention interaction term (Aiken \& West, 1991); this variable entered the regression equation at Step 4.

Table 1 shows the results of these analyses. At Step 1 , baseline consumption emerged as a strong predictor of consumption at follow-up and explained $41 \%$ of the variance. The inclusion of goal intention strength at Step 2 did not improve the fit of the model, and baseline consumption remained the only significant predictor. At Step 3, implementation intentions produced a marginally significant increase in the variance explained in behavior $(\beta=-.15, p=.083)$. Most important, however, the Goal Intention Strength $\times$ Implementation Intention interaction was associated with a significant increment in the variance explained in behavior $\left(\Delta R^{2}=\right.$ $.04, p<.01)$. In the final equation, baseline consumption and the interaction term were the only significant predictors of behavior.

We decomposed the interaction term in the manner specified by Aiken and West (1991). In particular, simple slopes for implementation intentions were computed at three levels of goal intention: low $(M-1 S D)$, moderate $(M)$, and high $(M+1 S D)$. Findings showed that when participants had low or moderate goal intentions, then implementation intention formation was not associated with consumption at follow-up ( $B s=.39$ and -.69 , respectively, ns). However, when participants' intention to reduce their snack food consumption was high, then implementation intention formation was associated with reduced consumption of participants' chosen snack food $(B=-1.76, p<.05)$. Thus, forming an implementation intention was particularly beneficial in reducing snack food consumption among participants who strongly intended to decrease their intake of snacks.

\section{Discussion}

Implementation intentions that specify thinking about a tempting food in the if-component and ignoring that thought in the then-component effectively shielded goal striving to halve respective food intake. This finding goes beyond past research on implementation intentions (see meta-analysis by Gollwitzer \& Sheeran, 2006) as it specifies a negative inner state (i.e., a critical thought), whereas most prior research specified external cues (i.e., a critical external context). Most important, the present study focused on shielding ongoing goal striving from getting derailed by disruptive thoughts (i.e., craving for high-fat snacks), whereas past research on implementation intentions focused on getting on with goal attainment by initiating goal-directed behaviors.

Study 1 drew upon a recent theory of desire (EITD; Kavanagh et al., 2005) to specify what if- and thencomponents of an implementation intention would be effective in reducing desire thoughts-and thereby promote the achievement of the goal of reducing consumption of high-fat snacks. According to the EITD, 
attending to and/or elaborating desire-related thoughts increases desire persistence and the likelihood of consequent consumption of snacks. The implementation intention therefore specified thoughts about snack food in the if-component of the plan as a negative inner state and specified an ignore response in the plan's then-component.

The obtained evidence supports the idea that controlling desire thoughts via implementation intentions is an effective strategy of reducing snack food consumption in two ways. First, implementation intention participants exhibited a sharper decline in consumption of snacks in a 1-week period compared to control participants. Second, control participants still ate significantly more than one half of their baseline consumption during the 1-week follow-up, whereas implementation intention participants reached the set standard-there was no difference between their consumption at followup and half of their baseline consumption.

Finally, as was also found with implementation intention effects on action initiation with respect to the strength of participants' underlying goal intentions (e.g., Sheeran et al., 2005), snack food consumption was least when participants both strongly intended to reduce consumption and had formed if-then plans. Apparently, action control by implementation intentions flexibly respects the strength of the superordinate goal no matter whether the goal-striving problem of getting started or staying on track is at issue.

In sum, the results suggest that implementation intentions that specify desire thoughts (i.e., a negative inner state) in the if-component and an ignore-response in the then-component help people to stay on track with self-improvement goals such as reducing the intake of unhealthy foods.

\section{STUDY 2: CONTROLLING INNER STATES THAT IMPAIR SPORTS PERFORMANCE}

Studies on sports competitions discovered that negative inner states (e.g., performance anxiety [Covassin \& Pero, 2004; Williams et al., 2002]; anger [Collins, 1991; Wughalter \& Gondola, 1991]; feelings of stress [Hanegby \& Tenenbaum, 2001]) hamper the quality of athletic performance. This is particularly true for sports that involve a direct competition with an opponent as it is the case with tennis and table tennis. We wondered whether specifying these negative inner states as critical cues in the if-component of implementation intentions and a goal-shielding response in the then-component supports staying on track under such adverse inner states. The specified goal-shielding responses were taken from research that analyzed strategies of improving one's performance during a tennis match (e.g., ignoring the noise made by the audience, focusing one's attention on the ball, engaging in relaxation behavior; see Anshel \& Anderson, 2002; Mamassis \& Dogamis, 2004; Ryska, 1998).

In the present study, we manipulated participants' goal intention to perform well in a tennis match rather than simply measuring it. Accordingly, we had a nogoal control condition and a mere goal intention condition in which tennis players the day before a critical match were assigned the following goal: "I will play each ball with utmost concentration and effort in order to win the match!" In the implementation intention condition, participants were also assigned this goal, and in addition, were asked to form four if-then plans that specified internal states in the if-components (e.g., performance anxiety, physiological arousal) and stayingon-track responses in the then-components (e.g., ignoring the adverse inner state).

\section{Method}

\section{Participants}

One hundred seven tennis players took part in the study (76 men and 31 women; age ranged from 14 to 68 years, with an average age of 34 years). All players regularly took part in tournaments of different German leagues. These leagues are grouped into 13 levels of performance excellence $(1=$ worst, $13=$ best $)$, and participants in the present study were playing in one of the leagues ranked from 1 to 11 . In exchange for their participation, participants received a pair of tennis socks worth 5 Euros.

\section{Design}

Participants were randomly assigned to one of the three conditions: no goal versus goal intention versus implementation intention. As dependent variables we measured how participants and their trainers/teammates rated their performance and physical fitness during the match compared to former matches. Trainers and teammates were familiar with players' performance for at least 2 years.

\section{Procedure}

Some weeks before the match, participants were asked if they would like to take part in a study on athletic sports competitions. One day before the match, the experimenter visited the participant at home and introduced him or her to the study. All participants completed a questionnaire asking for background information (e.g., age, rank in league) and other information possibly 
important for the next day's performance (e.g., how fit they felt, how they evaluated their upcoming opponent's tennis ability). Participants in the goal intention and in the implementation intention then went through the procedures described below. Participants in the nogoal condition only completed the questionnaire about background and match-relevant information.

Next day prior to the match, the experimenter reminded participants of their intentions (goal intention and implementation intention condition) or just asked participants how they felt today (no-goal condition). Furthermore, he asked participants' trainers or teammates if they would be so kind as to assess the participant's performance for a sport psychology study. Immediately after the match, participants and their trainers/teammates rated the demonstrated performance and physical fitness compared to former matches. Finally, participants received a pair of tennis socks in exchange for their participation and were then carefully debriefed and thanked.

Background information questionnaire. The questionnaire was designed to investigate whether participants in the three experimental conditions differed with respect to gender, rank of league, rank position within the league, the number of years they had played in the current league, the number of years they had been taking part in tennis competitions, how well they coped with defeats, and whether the critical match was a home match or not. The questionnaire also included items that measured the selfrated probability of winning the critical match, self-rated capability to cope with difficult situations, knowledge of the opponent, self-rated ability compared to their upcoming opponent, and self-rated overall fitness. Finally, we asked participants for the number of victories and defeats in their former matches and measured their performance anxiety in competitions by means of the Sports Competition Anxiety Test (SCAT; Martens, 1977).

Goal intention manipulation. Participants were assigned the goal "I will play each ball with utmost concentration and effort in order to win the match!" Participants were handed this goal intention on a sheet of paper and were asked to endorse it by underlining it. Participants also were requested to write down their goal intention on a separate sheet of paper and to repeat it to themselves by inner speech next day prior to the match.

Implementation intention manipulation. Participants in the implementation intention condition were first assigned the above goal intention. Thereafter, they were asked to form implementation intentions by selecting 4 negative inner states from a list of 18 options provided. Participants were asked to choose those negative inner states from the list that occurred most frequently and had the most detrimental effect on their performance during tennis matches. The 18 options could be categorized into negative cognitive (e.g., "not concentrating enough"), motivational (e.g., "feeling self-abandoned"), physiological (e.g., "feeling exhausted"), and emotional inner states ("feeling angry"). Next, participants were asked to identify responses that were suitable for controlling the selected negative inner states. For this purpose, a list of 102 coping responses known to be effective during sports competition were presented (e.g., "... then I will risk something and play courageously!”, “. . . then I will calm myself and tell myself 'I will win!"”; e.g., Anshel \& Anderson, 2002; Mamassis \& Dogamis, 2004; Ryska, 1998). By selecting negative inner states and coping behaviors on their own, participants could compose their if-then plans in an individualized manner, thereby specifying their most personally relevant negative inner states (in the if-component of their plans) and linking them to the most appropriate goal-directed responses (in the thencomponent of their plans). Altogether, each participant arrived at four individually chosen if-then plans that had to be written down on a sheet of paper. We did this to facilitate participants' endorsement of the composed implementation intentions.

Self-assessment of performance and physical fitness. After the critical match, participants rated their performance and physical fitness during the match compared to former matches on visual analogue scales ranging from 0 (worse) to $5.55 \mathrm{~cm}$ (better).

Assessment of players' performance and physical fitness by trainers/teammates. After the match, participants' performance and fitness was rated by their trainers (or by teammates if the trainer was not present at the match) on equivalent scales to those used for self-assessments.

Manipulation check. After the match, participants in the goal intention and in the implementation intention condition were asked if they had set themselves the goal intention "I will play each ball with utmost concentration and effort in order to win the match tomorrow!" prior to the match (yes-no), how strongly they felt committed to this goal $(0-5.55 \mathrm{~cm}$; reaching from not at all to very), and how important this goal had been for them during the match $(0-5.55 \mathrm{~cm}$, reaching from not at all to very).

\section{Results}

\section{Equivalence of Conditions}

Chi-square-tests, ANOVAS, and planned contrasts were used to compare the three different conditions on 
the questionnaire items assessing background and match-relevant information. The three groups did not differ with respect to gender, rank of league, rank position within the league, number of years they had played in the current league, number of years they had been taking part in tennis competitions, how well they coped with defeats, and whether the critical match was a home match or not (all $F s<1$; all $\chi^{2} s<1$ ). There were also no differences between conditions on self-rated probability of winning the critical match, self-rated capability to cope with difficult situations, knowledge of the opponent, self-rated capability of playing tennis compared to their upcoming opponent, self-rated overall fitness, or the number of victories and defeats in former matches during the current season (all Fs $<1$ ). The age of the participants tended to be different, $F(2,104)=2.57, p=.08$. Participants in the implementation intention condition $(M=$ $39, S D=15)$ were older than participants in the goal intention condition $(M=32, S D=13), t(73)=2.02, p<$ .05 , and tended to be older than participants in the nogoal condition $(M=32, S D=14), t(67)=1.82, p=.07$.

Analyzing the SCAT (Martens, 1977), we discovered a marginal difference between conditions, $F(2,104)=$ $2.94, p=.05$. Participants in the implementation intention condition $(M=20.19, S D=5.98)$ reported more anxiety in sport competitions than participants in the goal intention condition $(M=17.42, S D=5.00)$, $t(73)=2.18, p<.05$, but not compared to participants in the control condition $(M=19.72, S D=4.68), t<1$.

Finally, an analysis of how long trainers/teammates who evaluated participants' performance and physical fitness knew the participant and how familiar they felt with his or her performance and fitness revealed no significant differences between the conditions $\left(F_{s}<1\right)$.

\section{Manipulation Checks}

As expected, the one-factorial ANOVA on "How strongly were you committed to your goal?" was not significant (goal intention condition: $M=4.09, S D=1.44$; implementation intention condition: $M=4.23, S D=1.34$; $F<1)$. Thus, forming the implementation intentions did not enhance commitment to the goal intention, which rules out this variable as a potential explanation of any implementation intention effects. Furthermore, there was no significant difference between conditions in how important it was for participants to pursue their goal (goal intention condition: $M=4.45, S D=0.96$; implementation intention condition: $M=4.39, S D=1.05 ; F<1$ ).

\section{Ratings of Physical Fitness and Performance in the Critical Match}

Self-ratings and other-ratings of performance and physical fitness were $z$-standardized and submitted to a 3

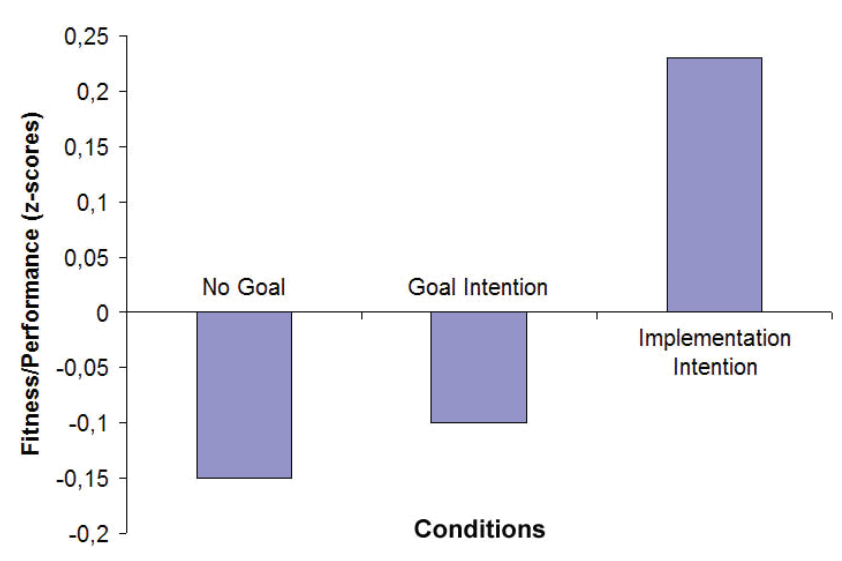

Figure 2 Physical fitness and performance compared to former matches by intention condition.

(Intention: No Goal vs. Goal Intention vs. Implementation Intention) $\times 2$ (Rating: Self vs. Other) $\times 2$ (Outcome: Physical Fitness vs. Performance) ANOVA, using the first factor as a between-participants factor and the last two factors as within-participant factors. This ANOVA revealed a significant main effect of Intention, $F(2,104)=4.24$, $p<.05$; no other significant main or interaction effects were observed. A post hoc least significant difference (LSD) test showed that there was no difference between the fitness and performance ratings in the no-goal $(M=-0.15$, $S D=0.12)$ and the goal intention condition $(M=-0.10$, $S D=0.10), p=.73$. However, the LSD test revealed significant differences between the no-goal $(M=-0.15$, $S D=0.12)$ and the implementation intention condition $(M=0.23, S D=0.10), p<.01$, and between the goal intention $(M=-0.10, S D=0.10)$ and the implementation intention condition $(M=0.23, S D=0.10), p<.05$. Thus, participants in the implementation intention condition rated themselves better on physical fitness and performance in the critical match and were rated better on these dimensions by trainers/teammates compared to participants in both the no-goal and goal intention conditions (Figure 2).

\section{Discussion}

In Study 2 we investigated whether implementation intentions can be used to shield goal striving during a tennis competition from negative inner states (e.g., anxiety, nervousness, lack of concentration). In this real-life setting in which a strong pressure to perform well predominates, it was observed that self- and other-ratings (trainers and teammates) of physical fitness and performance were higher for implementation intention participants as compared to both goal intention and no-goal participants. It is important to note that neither the participants nor the trainers/teammates knew the condition 
to which participants were assigned and therefore were blind to our hypothesis. These considerations seem to rule out participants' expectations or experimenter demand as an explanation of our findings.

Study 2 again demonstrated that negative inner states can successfully be used as initiating conditions for goaldirected responses specified in implementation intentions. Apparently, the use of implementation intentions can be expanded from spelling out external cues (e.g., particular locations and times) in the if-component of implementation intentions to specifying inner states that hold the potential to drive goal striving off-track. Goal-directed responses that are geared at alleviating the disruptive influence of these states (e.g., relaxation techniques, focusing one's concentration, self-regulation of emotions) can then be specified in the then-component of the implementation intention. The consequence is that goal-shielding responses can be triggered by internal cues and thereby increase performance in competition situations.

The results of Study 2 are impressive considering that participants in the implementation intention condition reported significantly greater anxiety during sport competitions than participants in the goal intention condition in the background questionnaire that was completed 1 day before the match. Thus, participants in the implementation intention condition performed very well and showed strong physical fitness, despite their high performance anxiety during sport competitions. In this sense, Study 2 also indicates that forming implementation intentions helps to shield goal striving from anxiety-related inner states.

Finally, Study 2 is one of the few implementation intention studies (e.g., Murgraff, White, \& Phillips, 1996) in which participants could choose their implementation intentions in an individualized manner and make use of more than just one implementation intention. Participants selected the four, most personally relevant, negative inner states as if-components of their implementation intentions and the four goal-directed responses that they assessed as being suited best for shielding their goal striving. These findings speak to the idea that implementation intention inductions can easily be tailored to the particular selfregulatory problems at hand (Gollwitzer \& Sheeran, 2006). To facilitate goal striving and increase rates of goal attainment, people can form multiple if-then plans, geared at alleviating the particular inner states they find most detrimental to their performance, and using the responses they perceive as most effective in shielding their goal.

\section{GENERAL DISCUSSION}

Two studies investigated the possibility of using negative inner states (i.e., cravings for fast food in Study 1 and states that hamper tennis performance in Study 2) as the if-components of implementation intentions and responses to those states in the plan's then-components to shield goal striving. Previous implementation intention research predominantly has been concerned with whether implementation intentions facilitate the initiation of goal-directed behavior under conditions in which action initiation is difficult (for review, Gollwitzer \& Sheeran, 2006). However, many goals require repeated and persistent striving to be accomplished and thus may present problems not only to do with initiating goal striving but also in keeping such striving on track. Accordingly, the present studies tested whether implementation intentions can also be used to shield ongoing goal pursuits from disruptive inner states.

Both studies were conducted in field settings and concerned important personal goals whose accomplishment relies on frequent and/or determined striving, namely, healthy eating (Study 1) and performance in a tennis competition (Study 2). In both studies, rates of goal attainment were enhanced by forming implementation intentions geared at shielding the superordinate goal from negative inner states. That is, an if-then plan focused on preventing elaboration of desire thoughts engendered a significant reduction in snack food consumption, whereas if-then plans that specified effective coping responses to cognitive, motivational, and emotional barriers to playing good tennis enhanced ratings of match fitness and performance. These findings extend previous research on implementation intentions by suggesting that if-then plan formation can be used not only to get started with goal striving but also to prevent goal striving from getting derailed by negative inner states.

One may ask whether the implementation intentions in the present studies only initiated goal-directed behavior at the beginning of reducing snack food consumption (Study 1) and the tennis match (Study 2) and thus do not produce permanent goal shielding. However, as goal striving in both studies aimed at an increase in performance across quite extended time intervals (i.e., 1 week in Study 1, and 1 to 5 hours in Study 2), it seems unlikely that the observed increase in performance in the implementation intention condition of both studies was only due to coping responses triggered at the beginning of goal striving. Rather, we assume that these coping responses were triggered repeatedly throughout the course of goal striving whenever the detrimental inner state specified in the if-component originated. Implementation intentions geared at the facilitation of the initiation of goal-directed action, on the other hand, operate quite differently in this respect. They specify good opportunities to get started with goal striving by executing an instrumental goal-directed response and thus should leave the course of subsequent goal striving unaffected. 


\section{Specifying Inner States Versus External Events}

Moreover, in the present studies, the goal-directed responses specified in the then-component of the implementation intentions studied were coping responses linked to various handicaps and weaknesses originating inside the person and not to challenges arising from outside the person. One therefore may wonder whether ifthen plans that link coping responses to disruptive external events will also be effective in shielding an ongoing goal pursuit. Research by Gollwitzer and Schaal (1998) on resistance to temptations provides an affirmative answer to this question, given that certain coping responses are specified in the then-component (e.g., ignore responses). Participants who had to perform arithmetic problems for a period of 15 minutes were more successful in doing so despite the presentation of various interspersed attractive video clips, when participants had formed implementation intentions that specified "attractive video clips" in the if-component and an "ignore" response in the then-component.

Reflecting on the pros and cons of forming implementation intentions that link a coping response to negative external events versus detrimental inner states, the following should be kept in mind: Specifying inner states has the advantage that these detrimental states could function as a summary label for all of those negative external events that might compromise goal striving-even those one is not aware of or may fail to anticipate. Accordingly, whenever a person is not in a good position to know about and anticipate critical events, specifying detrimental inner states seems to be the safer strategy to shield one's goal striving. For instance, patients with panic attacks are usually not aware of which kinds of external events trigger the attack and whether these events are to be expected in a forthcoming external context (e.g., Hinton, Nathan, \& Bird, 2002). For these patients, it would make sense to specify inner states (i.e., upcoming anxiety) as if-components in order to control their negative emotions. Another example is ADHD children who are not aware of the external cues that trigger their problematic behavior. These children could form implementation intentions that specify negative inner states (e.g., a feeling of irritation) and link them to instrumental coping responses (e.g., to concentrate on the ongoing task performance).

Finally, there is the question of whether it is better for effective goal shielding to specify inner states or external events in the if-components of one's implementation intentions. As said above, if potential critical events are known and can be correctly anticipated, a person may resort to specifying these events and thus does not have to specify inner states. In this case, whether it makes more sense to use the one strategy of forming implementation intentions or the other could depend on certain personality traits. For instance, people with a high capability for self-reflection might benefit more from specifying inner states as compared to external-events people, whereas people with low selfreflection might benefit more from specifying external events as compared to inner states. Moreover, there may be certain types of goals that benefit more from specifying inner states as compared to external events, and vice versa. For instance, it seems possible that internalstate implementation intentions are particularly effective in shielding abstinence goals from the intense cravings documented for substances such as tobacco, alcohol, or illicit drugs (Kavanagh et al., 2005) or protect (for instance) relationship or negotiation goals from the effects of "hot" emotions such as jealousy or anger (cf. Metcalfe \& Mischel, 1999), whereas external-event implementation intentions are particularly effective in shielding performance goals from disruptions (e.g., finish writing a manuscript from social disruptions). The issue of what type of coping response should be specified to deal with a certain type of negative inner state (e.g., an ignore response to craving thoughts, a calming response to the experience of agitation, a reappraisal response to dejection cues) and external events (e.g., a temptationinhibiting response or a task-focusing response) also requires careful explication to optimize if-then planning effects in preventing goal derailment. Finally, several individual-difference variables could potentially make goal shielding by means of implementation intentions more necessary or advantageous (e.g., high scores on sensation-seeking scales, low scores on coping or affect regulation strategies). Thus, there are a variety of directions that could be taken in further studies on implementation intentions and the shielding of goal striving.

\section{Potential Process Accounts}

In the present studies, we did not investigate the processes on which the effects of implementation intentions were based. Therefore, one can only speculate which kinds of processes might be instigated by specifying inner states in if-components of implementation intentions and linking them to coping responses geared at goal shielding. Previous implementation intention research observed that specifying external situations as if-components of implementation intentions and linking them to a response that initiates goal striving leads to a heightened cognitive accessibility of the external cue. As a consequence, the external cue is easily detected, readily attended to, and more successfully remembered (e.g., Aarts et al., 1999; Achtziger et al., 2007; Webb \& Sheeran, 2004; see Gollwitzer \& Sheeran, 2006). In line with these findings, it can be assumed that specifying 
detrimental inner states as if-components in implementation intentions should lead to a faster detection of these specified inner states compared to other inner states. Referring to the responses specified in the thencomponent, past research on implementation intentions observed the automation of action initiation-immediate and efficient initiation of the specified response without further conscious intent (e.g., Bayer et al., 2007; Brandstätter et al., 2001; Gollwitzer \& Brandstätter, 1997). As in Study 2 (i.e., during the tennis match), participants had to respond very fast to their inner states. Thus, not having time for resource-consuming effortful action initiation, it seems very likely that the coping responses specified in the implementation intentions of the present studies also became automated. Whether this automation of the coping responses generally leads to a reduced phenomenological experience of the specified detrimental inner states or even to a reduction of their physiological concomitants is up to future research. Such research might also address the question of whether it is the modification of these inner states that leads to better goal shielding or a stronger focus on the ongoing goal striving, or both.

\section{Related Research on Goal Shielding}

There are further approaches to goal shielding reported in the literature. Shah, Friedman, and Kruglanski (2002) provided the first evidence that goal activation automatically reduces the accessibility of other, alternative goals. Participants who were subliminally primed with words related to a personal goal were slower to respond to words describing other personal goals in a lexical decision task. Shah et al. also identified several goal characteristics (e.g., level of goal commitment, substitutive relations between goals) and motivational factors (e.g., need for closure, self-rated tenacity of goal striving) that intensified the extent to which the focal goal inhibited alternative goals (see also Shah \& Kruglanski, 2002). Fishbach, Friedman, and Kruglanski (2003) examined relations between goals and temptations (enjoyable activities that are perceived as antithetical to successful striving for the focal goal). Findings showed that temptations increased the accessibility of goal representations, even under conditions of high cognitive load, and that successful self-regulators were characterized by stronger temptation-goal associations compared to less successful self-regulators. The work of Mischel and colleagues (e.g., Mischel \& Ebbesen, 1970; Peake, Hebl, \& Mischel, 2002) on delay of gratification is also relevant. It describes the pursuit of more attractive but temporally distal outcomes in the face of immediately available but less desirable rewards. It is observed that people who were good self-regulators in childhood later in their lives turn out to be more cognitively skilled, more socially competent, and better able to cope with stress than others. Baumeister and colleagues (e.g., Baumeister, Bratlavsky, Muraven, \& Tice, 1998) repeatedly demonstrated that one act of volition has detrimental impact on subsequent goal shielding. Resisting temptations, for instance, undermines self-regulation in a subsequent, unrelated domain (e.g., persistence at a frustrating task). And finally, Trope and Fishbach (2000) report evidence for counteractive control geared at shielding one's goals; this is a readiness to engage in proactive bolstering of the value of a preferred activity to the extent that the activity is associated with short-term costs.

The present research expands these analyses of shielding goals from temptations (e.g., Mischel \& Ebbesen, 1970; Peake et al., 2002), alternative goal pursuits (Shah et al., 2002), ego depletion (e.g., Baumeister et al., 1998), and counteractive control (Trope \& Fishbach, 2000) by examining the issue of protecting goal striving from antagonistic thoughts and feelings. Our findings indicate that negative inner states such as craving thoughts, nervousness, or lack of concentration can also imperil an ongoing goal pursuit and may need to be guarded against to successfully reach one's goals (see also Tice, Bratslavsky, \& Baumeister, 2001). As the present two studies are the first experiments on specifying inner states in the if-components of implementation intentions and linking them to coping responses that shield the ongoing goal striving, it is not surprising that questions remain about how and why these implementation intentions worked. For instance, we do not know yet whether the experience specified inner states and their physiological concomitants become less pronounced by being linked to coping responses in if-then plans or whether it is only that such if-then plans facilitate the allocation of effort to the ongoing goal pursuit rather than suppressing the inner state. Future research should investigate these possible underlying mechanisms of if-then plans that link detrimental inner states to coping responses, thus trying to protect an ongoing goal striving form getting derailed.

\section{Applied Implications}

The present studies provide novel evidence that mere commitment to one's overarching goal may not necessarily be effective in warding off disruptive thoughts and feelings. For instance, the strength of dieting-goal intentions did not predict subsequent snack food consumption in Study 1, and adopting the goal of playing every ball with the utmost concentration and effort did not improve tennis performance compared to not adopting that goal in Study 2. Rather, both studies 
showed that it was necessary to furnish one's goal intentions with respective implementation intentions in order to successfully shield goal striving from the detrimental effects of negative inner states.

Two particular advantages of using implementation intentions for goal shielding are easy applicability and adaptability of this self-regulatory tool. Implementation intentions can be formed on the spot (in Study 2, participants did not find it hard to come up with writing if-then plans on their own), and they do not need much cognitive elaboration (in Study 1, a simple ignore response sufficed). Thus, individuals can tailor their if-then plans to the negative inner states that strike them to be problematic for their ongoing goal pursuits, and if need be, can form multiple if-then plans to deal with a variety of disruptive self-states. Moreover, by specifying negative thoughts or feelings in the if-component of the plan, the person is well equipped to deal with numerous situations that might give rise to these inner states and thus does not need to plan how to deal with each particular situation in advance. In sum, implementation intentions appear to be an effective tool for shielding superordinate goals because respective plans can be formed when, how often, and by whom they are needed.

\section{REFERENCES}

Aarts, H., Dijksterhuis, A., \& Midden, C. (1999). To plan or not to plan? Goal achievement or interrupting the performance of mundane behaviors. European Journal of Social Psychology, 29, 971-979.

Achtziger, A., Bayer, U. C., \& Gollwitzer, P. M. (2007). Implementation intentions: Increasing the accessibility of cues for action. Unpublished manuscript, University of Konstanz, Gemany.

Achtziger, A., \& Gollwitzer, P. M. (2008). Motivation and volition in the course of action. In J. Heckhausen \& H. Heckhausen (Eds.), Motivation and action (pp. PAGES. Cambridge, UK: Cambridge University Press.

Aiken, L. S., \& West, S. G. (1991). Multiple regression: Testing and interpreting interactions. Newbury Park, CA: Sage.

Ajzen, I. (1991). The theory of planned behavior. Organizational Behavior and Human Decision Processes, 50, 179-211.

Anshel, M. H., \& Anderson, D. I. (2002). Coping with acute stress in sport: Linking athletes' coping style, coping strategies, affect, and motor performance. Anxiety, Stress o Coping: An International Journal, 15, 193-209.

Baumeister, R., Bratlavsky, E., Muraven, M., \& Tice, D. M. (1998). Ego depletion: Is the active self a limited resource? Journal of Personality and Social Psychology, 74, 1252-1265.

Bayer, U. C., Achtziger, A., Malzacher, J. T., Moskowitz, G., \& Gollwitzer, P. M. (2007). Responding to subliminal cues: Do ifthen plans cause action preparation and initiation without conscious intent? Manuscript under review.

Bayer, U. C., \& Gollwitzer, P. M. (2007). Boosting scholastic test scores by will power: The role of implementation intentions. Self and Identity, 6, 1-19.

Brandstätter, V., Lengfelder, A., \& Gollwitzer, P. M. (2001). Implementation intentions and efficient action initiation. Journal of Personality and Social Psychology, 81, 946-960.

Collins, S. W. (1991). The role of anger among male college level tennis players. Dissertation Abstracts International, 52(5-B), 2769.
Covassin, T., \& Pero, S. (2004). The relationship between self-confidence, mood state, and anxiety among collegiate tennis players. Journal of Sport Behavior, 27, 230-242.

Crowne, D. P., \& Marlowe, D. (1960). A new scale of social desirability independent of psychopathology. Journal of Consulting Psychology, 24, 349-354.

Fishbach, A., Friedman, R. S., \& Kruglanski, A. W. (2003). Leading us to not unto temptation: Momentary allurements elicit overriding goal activation. Journal of Personality and Social Psychology, 84, 296-309.

Garner, D. M., \& Wooley, S. C. (1991). Confronting the failure of behavioral and dietary treatments of obesity. Clinical Psychology Review, 6, 58-137.

Gollwitzer, P. M. (1993). Goal achievement: The role of intentions. European Review of Social Psychology, 4, 141-185.

Gollwitzer, P. M. (1999). Implementation intentions: Strong effects of simple plans. American Psychologist, 54, 493-503.

Gollwitzer, P. M., Bayer, U. C., \& McCulloch, C. (2005). The control of the unwanted. In R. Hassin, J. Uleman, \& J. A. Bargh (Eds.), The new unconsciousness (pp. 485-515). Oxford, UK: Oxford University Press.

Gollwitzer, P. M., \& Brandstätter, V. (1997). Implementation intentions and effective goal pursuit. Journal of Personality and Social Psychology, 73, 186-199.

Gollwitzer, P. M., \& Schaal, B. (1998). Metacognition in action: The importance of implementation intentions. Personality and Social Psychology Review, 2, 124-136.

Gollwitzer, P. M., \& Sheeran, P. (2006). Implementation intentions and goal achievement: A meta-analysis of effects and processes. Advances in Experimental Social Psychology, 38, 69-119.

Hanegby, R., \& Tenenbaum, G. (2001). Blame it on the racket: Norm-breaking behaviors among junior tennis players. Psychology of Sport and Exercise, 2, 117-134.

Heatherton, T. F., Mahamedi, F., Striepe, M., Field, A. E., \& Keel, P. (1997). A 10-year longitudinal study of body weight, dieting, and eating disorder symptoms. Journal of Abnormal Psychology, 106, 117-125.

Herman, C. P., \& Polivy, J. (2004). The self-regulation of eating: Theoretical and practical problems. In R. F. Baumeister \& K. D. Vohs (Eds.), Handbook of self-regulation: Research, theory, and applications. New York: Guilford.

Hinton, D., Nathan, M., \& Bird, B. (2002). Panic probes and the identification of panic: A historical and cross-cultural perspective. Culture, Medicine, and Psychiatry, 26, 137-153.

Kavanagh, D. J., Andrade, J., \& May, J. (2005). Imaginary relish and exquisite torture: The elaborated intrusion theory of desire. Psychological Review, 112, 446-467.

Loewenstein, G. (1996). Out of control: Visceral influences on behavior. Organizational Behavior and Human Decision Processes, 65, 272-292.

Mamassis, G., \& Dogamis, G. (2004). The effects of a mental training program on juniors pre-competitive anxiety, self-confidence, and tennis performance. Journal of Applied Sport Psychology, 16, 118-137.

Martens, R. (1977). Sport Competition Anxiety Test. Champaign, IL: Human Kinetics.

Metcalfe, J., \& Mischel, W. (1999). A hot/cool-system analysis of delay of gratification: Dynamics of willpower. Psychological Bulletin, 106, 3-19.

Mischel, W., \& Ebbesen, E. B. (1970). Attention in delay of gratification. Journal of Personality and Social Psychology, 16, 329-337.

Murgraff, V., White, D., \& Phillips, K. (1996). Moderating binge drinking: It is possible to change behavior if you plan in advance. Alcohol and Alcoholism, 31, 577-582.

Peake, P. K., Hebl, M., \& Mischel, W. (2002). Strategic attention deployment for delay of gratification in working and waiting situations. Developmental Psychology, 38, 313-326.

Ryska, T. A. (1998). Cognitive-behavioral strategies and precompetitive anxiety among recreational athletes. Psychological Record, 48, 697-708.

Shah, J. Y., Friedman, R., \& Kruglanski, A. W. (2002). Forgetting all else: On the antecedents and consequences of goal shielding. Journal of Personality and Social Psychology, 83, 1261-1280. 
Shah, J. Y., \& Kruglanski, A. W. (2002). Priming against your will: How goal pursuit is affected by accessible alternatives. Journal of Experimental Social Psychology, 38, 369-383.

Sheeran, P., Webb, T. L., \& Gollwitzer, P. M. (2005). The interplay between goal intentions and implementation intentions. Personality and Social Psychology Bulletin, 31, 87-98.

Strahan, R., \& Gerbasi, K. C. (1972). Short, homogenous versions of the Marlowe-Crowne Social Desirability Scale. Journal of Clinical Psychology, 28, 191-193.

Tice, D. M., Bratslavsky, E., \& Baumeister, R. F. (2001). Emotional distress regulation takes precedence over impulse control: If you feel bad, do it! Journal of Personality and Social Psychology, 80, 53-67.

Trope, Y., \& Fishbach, A. (2000). Counteractive self-control in overcoming temptation. Journal of Personality and Social Psychology, 79, 493-506.
Webb, T. L., \& Sheeran, P. (2004). Identifying good opportunities to act: Implementation intentions and cue discrimination. European Journal of Social Psychology, 34, 407-419.

Williams, A. M., Vickers, J., \& Rodrigues, S. (2002). The effects of anxiety on visual search, movement kinematics, and performance in table tennis: A test of Eysenck and Calvo's processing efficiency theory. Journal of Sport \& Exercise Psychology, 24, 438-455.

Wughalter, E. H., \& Gondola, J. C. (1991). Mood states of professional female tennis players. Perceptual and Motor Skills, 73, 187-190.

Received March 29, 2007

Revision accepted August 29, 2007 